\title{
Fate of the Atrioventricular Endocardial Cushions in the Developing Chick Heart
}

\author{
CLIFFORD CHIN, REGINA GANDOUR-EDWARDS, SHARON OLTJEN, AND MICHAEL CHOY \\ Division of Pediatric Cardiology [C.C., S.O., M.C.] and Department of Pathology [R.G.J, University of California \\ Davis Medical Center, Sacramento, California 95817
}

\begin{abstract}
To determine the fate of the atrioventricular endocardial cushions in cardiac development, we used staining methods for extracellular fibronectin, which is abundant in the endocardial cushions, and actin, which is abundant in the myocytes. White Leghorn chick embryo hearts were harvested at Hamburger and Hamilton stages 26 to 36, and serial sections of the atrioventricular valve region were stained. Before atrioventricular valve formation, fibronectin and actin staining reveal separation between the fibronectin-rich endocardial cushions and the actin-rich myocardial layer. The developing mitral valve leaflets at all of the observed stages contain a fibronectinrich matrix but no actin-rich myocytes. In contrast, the tricuspid band includes both fibronectin matrix and actinrich cells. We conclude that the mitral valve leaflets in the chick form predominantly from the endocardial cushion tissue, and the tricuspid band receives contributions from both the endocardial cushions and surrounding myocardium. (Pediatr Res 32: 390-393, 1992)
\end{abstract}

The endocardial cushions are derived from transformed endothelial cells and cardiac jelly (1-3) sandwiched between the single layer of endothelial cells and the myocardium (4). At Hamburger and Hamilton stage 15 (5) of chick embryonic development, some of the endothelial cells transform into mesenchymal cells (fibroblasts) and migrate into the cardiac jelly, possibly under the influence of transforming growth factor- $\beta(6,7)$. The mesenchymal cells proliferate, produce an extracellular matrix rich in fibronectin, and become the major cellular component of the endocardial cushions (8). During early development, the endocardial cushions function as valves (9). They later fuse with the interatrial septum and the interventricular septum, dividing into distinct mitral and tricuspid valve components (10). The controversy begins at this point about the fate of the endocardial tissue.

This study was undertaken to determine the origin of the atrioventricular valves in the chick embryo. Using immunohistochemical staining, we showed that the mitral valve leaflets contain solely endocardial cushion tissue, whereas the tricuspid band includes both myocardial cells and endocardial cushion.

\section{MATERIALS AND METHODS}

Antibodies. Dr. E. Ruoslahti (La Jolla Cancer Research Foundation, La Jolla, CA) generously supplied the goat anti-chicken plasma fibronectin antibody used for fibronectin staining-a polyclonal antibody. A fluorescein-conjugated rabbit anti-goat IgG served as the second antibody and was obtained from ICN

Received February 27, 1992; accepted June 4, 1992.

Correspondence and reprint requests: Michael Choy, M.D., Division of Pediatric Cardiology. University of California Davis Medical Center, 2516 Stockton Blvd., Sacramento. CA 95817.

Supported by a grant-in-aid from the American Heart Association.
ImmunoBiologicals (Lisle, IL). Both the rabbit anti-actin antibody and fluorescein-conjugated goat anti-rabbit IgG were obtained from ICN ImmunoBiologicals for actin staining. The polyclonal anti-actin antibody was produced using isolated actin from chicken back muscle. It stains stress fibers of cultured chick fibroblasts and I bands of skeletal muscle.

Histology and immunohistochemical staining. White Leghorn chicken eggs were incubated at $37.5^{\circ} \mathrm{C}$ in humidified room air for $5,6,7,8,9$, and $10 \mathrm{~d}$, which produced embryos at Hamburger and Hamilton stages 26, 29, 31, 34, 35, and 36. The embryos were harvested in $0.9 \%$ normal saline, and the hearts were isolated and fixed in Carnoy's solution $(1.0 \mathrm{~mL}$ acetic acid, 6.0 $\mathrm{mL} 100 \%$ ethanol, and $3.0 \mathrm{~mL}$ chloroform). After embedding in paraffin, the hearts were sectioned in the frontal plane at a thickness of $6 \mu \mathrm{m}$. Serial sections were mounted on gelatincoated slides and stained as described below.

A standard immunofluorescence procedure was performed for extracellular fibronectin produced by the mesenchymal cells of the endocardial cushion (11). Similar sections were stained for intracellular actin to discriminate actin-rich myocytes from mesenchymal cells. Negative control sections were incubated in saline containing BSA, nonimmune goat serum, or nonimmune rabbit serum. Other serial sections were stained with a standard hematoxylin and eosin procedure for anatomy and cell death determinations. The sections were viewed and photographed using either a Zeiss WL or Leitz Dialux microscope, both equipped with epifluorescence.

\section{RESULTS}

The superior and inferior endocardial cushions are well developed by stage 26 in the chick, and a common atrioventricular valve orifice exists without identifiable mitral or tricuspid valves. The border between the endocardial cushions and the myocardial layer is easily distinguishable, and a few myocytes appear to migrate into the endocardial cushion (Figs. 1 and 2).

The atrioventricular valves begin to take shape at stage 31 , and the valves mature by restructuring of the endocardial cushion masses. On the mitral side, the valve leaflets form from the fibronectin-rich matrix tissue without contribution of the surrounding actin-rich cells. In the chick, the mature tricuspid valve is a muscle band instead of leaflets (12). The tricuspid band receives contributions from both the endocardial cushion tissue and ventricular myocardium (Figs. 3 and 4). The mature mitral and tricuspid valves are present by stages 35 and 36 , although some remodeling continues in additional stages. Again, only mesenchymal cells and an extracellular matrix compose the mitral valve leaflets, whereas myocytes contribute to the tricuspid band (Figs. 5 and 6). Survey of the hematoxylin and eosinstained sections of stages 26 through 36 chick reveals only rare dying cells and no phagocytic cells in the atrioventricular endocardial cushions and valve leaflets (Fig. 7). 

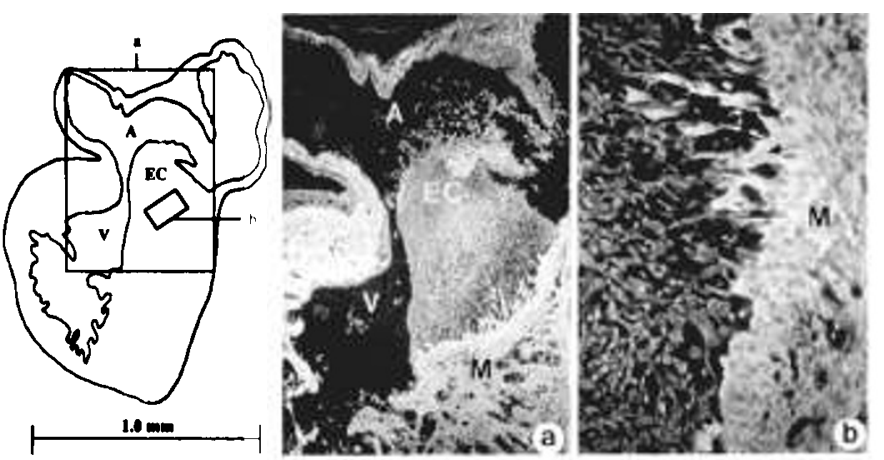

Fig. 1. Stage 26 chick heart stained for actin. The atrium $(A)$, ventricle $(V)$, and endocardial cushion $(E C)$ are labeled on the line diagram of the heart section. $a$, There is a common atrium with the inferior endocardial cushion tissue. The stain is specific for intracellular actin that highlights the myocytes $(M)$. The large but low-intensity staining material is the cushion tissue. $b$, A high-power view shows a clear line of demarcation between the low-intensity staining cushion tissue and the highintensity staining of the surrounding myocytes. High-intensity staining myocytes (black arrow) are migrating into the endocardial cushion tissue that will ultimately form part of the tricuspid band or base of the mitral valve leaflets.
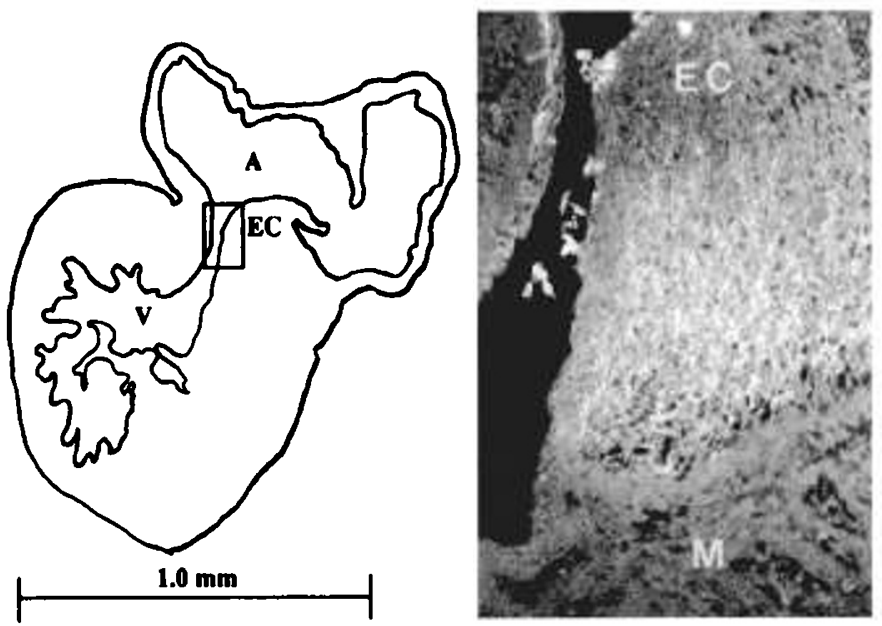

Fig. 2. Stage 26 chick heart stained for fibronectin. The inferior endocardial cushion with staining for extracellular fibronectin contrasts with the low-intensity staining of the surrounding myocytes. Abbreviations are the same as those in Figure 1. In stage 29 (not shown) the atrioventricular valve components have not developed yet, and staining for both actin and fibronectin show results similar to stage 26 .

\section{DISCUSSION}

In recent studies of the descriptive embryology of the human heart, some investigators believed that invagination and myocardial undermining result in the formation of the atrioventricular valves (13). The endocardial cushion tissue functions as a glue, holding together myocardial structures $(14,15)$ and does not materially contribute to the mitral valve except for a small portion of the aortic leaflet $(13,16,17)$.

Descriptive embryology using graphic reconstruction of tissue sections stained with hematoxylin and eosin has limitations, and several investigators have stated the difficulty in recognizing contributions of either ventral or dorsal cushion $(1,18)$. Cell labeling techniques have the advantage of tracing the migration of a small population of cells during the growth and remodeling of the endocardial cushions, but obviously these techniques cannot be applied to human embryos. De la Cruz et al. (18), using in vivo labeling techniques in the chick, showed that the basal and free portion of the anteroseptal leaflet of the mitral valve had origins from both endocardial cushions. Garcia-Pelaez
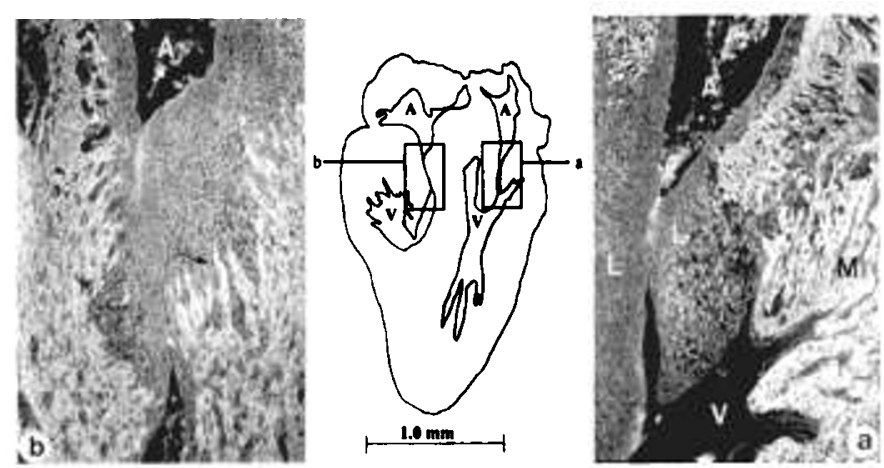

Fig. 3. Stage 31 chick heart stained for actin. $a$, The early mitral valve leaflets $(L)$ are visible as poorly stained structures next to the actinstained myocytes. Abbreviations are the same as those used in Figure 1. $b$, The tricuspid band region is forming from endocardial cushion with actin-rich cells (black arrow) invading the cushion tissue. With fibronectin staining (not shown), the mitral valve and tricuspid band consist of extracellular matrix rich in fibronectin, but staining in the myocyte region remains fibronectin-poor.
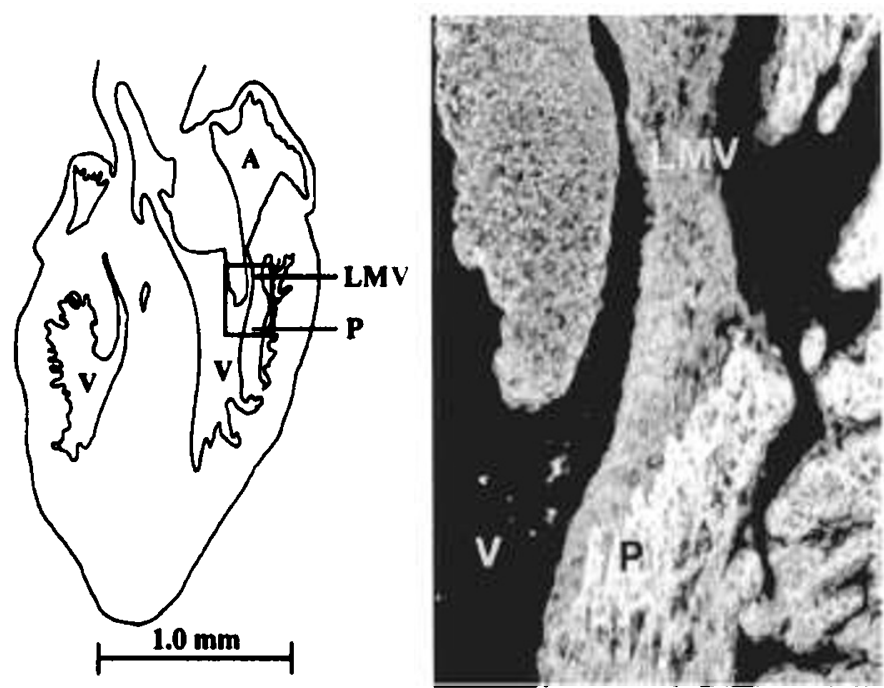

Fig. 4. Stage 34 chick heart stained for actin. The line diagram shows the lateral leaflet of the mitral valve ( $L M V)$ with its adjoining papillary muscle $(P)$. $A$, atrium; $V$, ventricle. The mitral valve continues to mature without material contribution of actin-rich myocytes. A papillary muscle composed of actin-rich myocytes reveals a clear line of separation from the actin-poor valvular tissue.
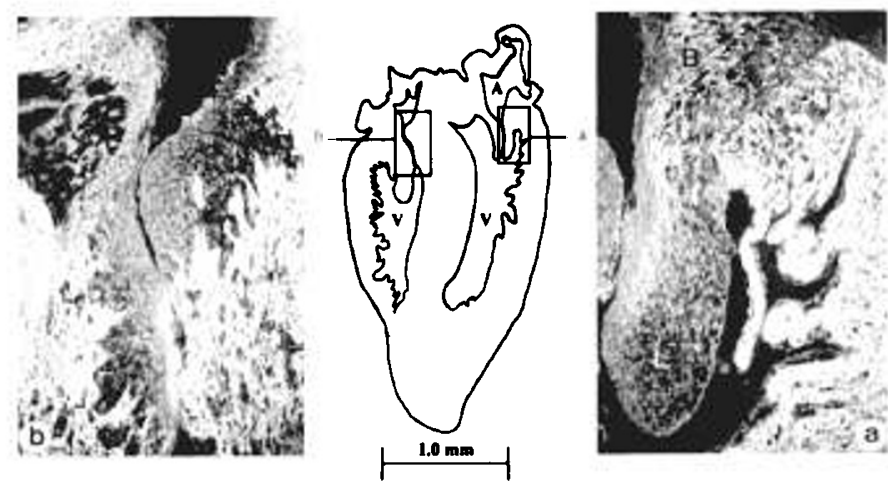

Fig. 5. Stage 35 chick heart stained for actin. $a$, The mitral valve is more mature. The mitral valve leaflet $(L)$ contains fibroblasts without any apparent myocytes, but the composition of the leaflet base $(B)$ is a mixture of myocytes and fibroblasts. $A$, atrium: $V$, ventricle. $b$, The tricuspid band is composed of both actin-rich and actin-poor cells. 


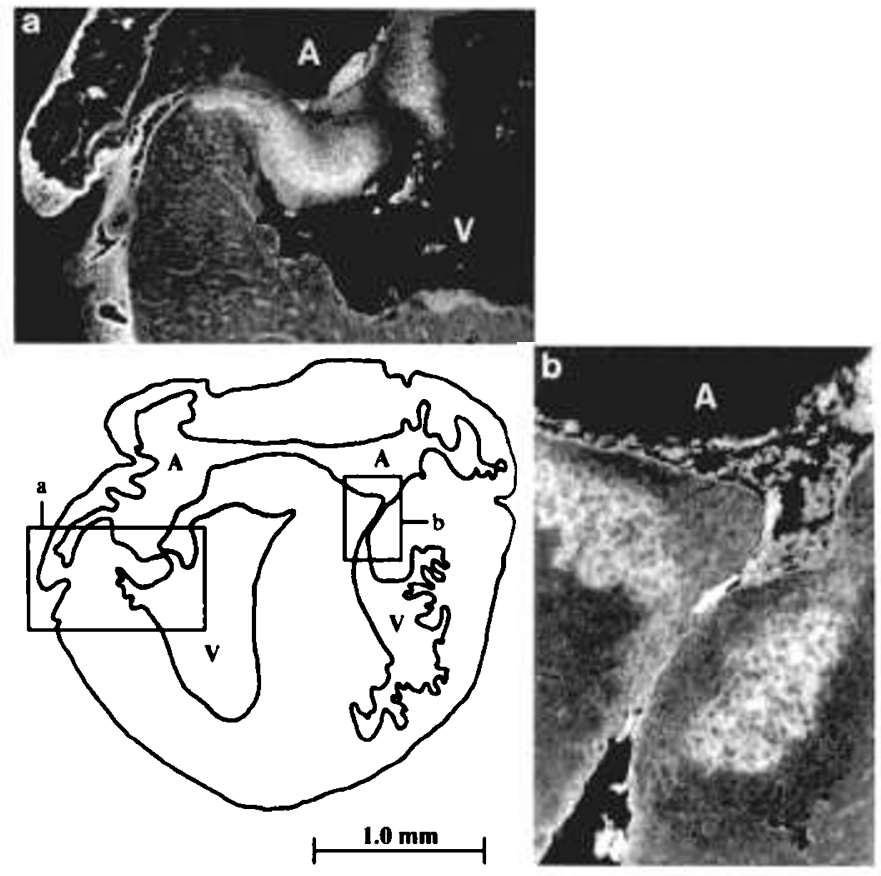

Fig. 6. Stage 36 chick heart stained for fibronectin. The mitral valve $(a)$ and tricuspid band $(b)$ are rich in extracellular fibronectin. $A$, atrium; $V$, ventricle. Actin and fibronectin staining in the earlier stage 35 heart (not shown) are identical with stage 36 .

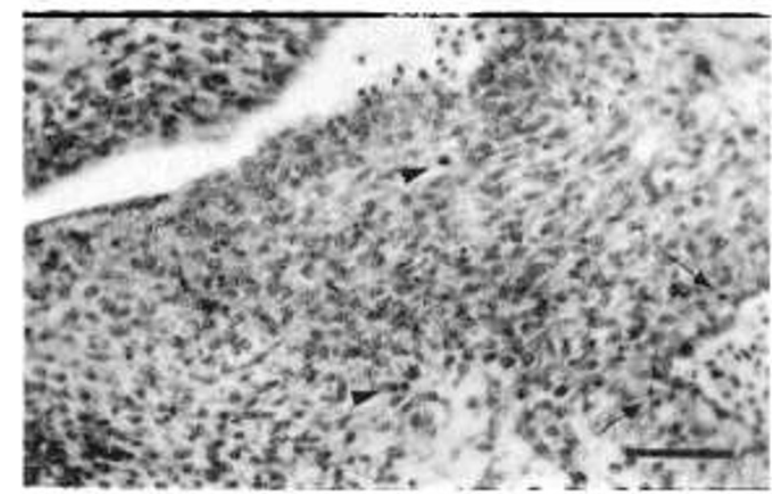

Fig. 7. Atrioventricular endocardial cushion of stage 29 chick heart stained with hematoxylin and eosin. The atrioventricular endocardial cushion shows a normal background of cell death of about $1 \%$ of the total number of cells (black triangles) and active cell mitosis (black arrows). Cell death zones as defined by Pexieder (26) and phagocytic cells were not detected. Bar $=0.05 \mathrm{~mm}$.

et al. (19) labeled the superior (ventral) endocardial cushion and found the marker not only in a large area of the left ventricular infundibulum, but also in the base of the free portion of the anteroseptal mitral leaflet.

We used staining techniques that would specifically stain certain tissue types of the developing chick heart and followed those tissues during development. Other investigators demonstrated that fibronectin is present in the cardiac jelly before and during mesenchymal migration in the endocardial cushions, but these studies did not reveal the origin of the fibronectin $(20,21)$. Ffrench-Constant and Hynes $(22,23)$ localized mRNA coding for fibronectin in the mesenchymal cells and endocardial cells of the stage 24 endocardial cushion using in situ hybridizations. In addition, the myocytes of the myocardium did not produce any of the fibronectin mRNA. Armstrong and Armstrong (24) established that mesenchymal cells of stage 36 hearts grown in monolayer culture produce a significant amount of extracellular fibronectin. Thus, we have strong evidence that the fibronectin stain- ing used in this study will specifically stain the extracellular matrix derived from mesenchymal tissue. It is clear from our data that a fibronectin-rich tissue makes up the atrioventricular endocardial cushions and continues to be present in both of the developing atrioventricular valves. Ruzicka and Schwartz (25) published a thorough investigation on the appearance of various actin mRNA isoforms in the chick embryonic heart using in situ hybridizations and dot blots. They provided no data suggesting that any of the forms exists in the atrioventricular endocardial cushion region only. Thus, the low-intensity actin staining of the endocardial cushions in this study is not due to the presence of myocytes with a form of actin unrecognized by the antibody. Clearly, the actin-rich cells intermingle with cells in the tricuspid band but not in the mitral leaflets. In conclusion, we agree with De la Cruz et al. (18) and Garcia-Pelaez et al. (19) that the endocardial cushions physically contribute to the atrioventricular valves.

We have considered another interpretation of our findings. Cells in the early endocardial cushions may die and be replaced with fibroblasts that originate from transformed myocytes of the surrounding myocardium. Pexieder (26) defined "cell death zones" or "cell death foci" that contain dying or dead cells in 30-70 volume percent of the tissue. He did find cell death zones in the atrioventricular cushions. Pexieder and other investigators have suggested other areas of programmed cell death such as the bulbar and truncal region in the developing heart (26-28). During our survey of atrioventricular valve development, we saw evidence of the normal background cell death that made up less than $3 \%$ by volume of the tissue, but we did not observe cell death zones or phagocytosis with hematoxylin and eosin staining. Fananapazir and Kaufman (29) also have questioned many of the original publications on cell death, since they observed no cell death zones in the bulbar cushion of the developing mouse heart. We did not try to establish whether myocytes transform into fibroblasts and migrate into the endocardial cushions, and there is very little literature on this topic. Arguello et al. in 1978 (30) provided some evidence of myocardial cell to fibroblast transformation in the truncal region of the chick heart.

In conclusion, using staining methods for extracellular fibronectin and intracellular actin, we have speculated on the origins of the atrioventricular valvular apparatuses. We suggest that the atrioventricular endocardial cushions are involved in the morphogenesis of both the mitral valve and tricuspid band in the chick. The mitral valve leaflets appear to be derived from endocardial tissue while the base of the valve leaflets is composed of a combination of fibroblasts and myocytes. The tricuspid band appears to have a dual origin, endocardial cushion tissue and myocardium.

\section{REFERENCES}

1. Los JA, van Eijndthoven E 1973 The fusion of the endocardial cushions in the heart of the chick embryo. Z Anat Entwicklungsgesch 141:55-75

2. Markwald RR, Fitzharris TP, Manasek FJ 1977 Structural development of the endocardial cushions. Am J Anat 148:85-120

3. Kinsella MG, Fitzharris TP 1980 Origin of cushion tissue in the developing chick heart: cinematographic recordings of in situ formation. Science 207:1359-1360

4. Hay DA, Low FN 1972 The fusion of dorsal and ventral endocardial cushions in the embryonic chick heart: a study in fine structure. Am J Anat 133:124

5. Hamburger V, Hamilton HL 1951 A series of normal stages in the development of the chick embryo. J Morphol 88:49-92

6. Potts JD, Runyan RB 1989 Epithelial-mesenchymal cell transformation in the embryonic heart can be mediated, in part, by transforming growth factor beta. Dev Biol 134:392-401

7. Potts JD, Dagle JM, Walder JA, Weeks DL, Runyan RB 1991 Epithelial mesenchymal transformation of embryonic cardiac endothelial cells is inhibited by a modified antisense oligodeoxynucleotide to transforming growth factor beta 3. Proc Natl Acad Sci USA 88:1516-1520

8. Choy M, Armstrong MT, Armstrong PB 1990 Regulation of proliferation of embryonic heart mesenchyme: role of transforming growth factor-beta $\perp$ and the interstitial matrix. Dev Biol 141:421-425

9. Van Gils FAW 1979 The development of the human atrioventricular heart valves. J Anat 128:427(abstr) 
10. Magovern JH, Moore GW, Hutchins GM 1986 Development of the atrioventricular valve region in the human embryo. Anat Rec 215:167-181

11. Choy M, Armstrong MT, Armstrong PB 1991 Transforming growth factorbeta 1 localized within the heart of the chick embryo. Anat Embryol (Berl) 183:345-352

12. Vassall-Adams PR 1982 The development of the atrioventricular bundle and its branches in the avian heart. J Anat 134:169-183

13. Wenink ACG, Gittenberger-de Groot AC 1986 Embryology of the mitral valve. Int J Cardiol 11:75-84

14. Wenink ACG, Gittenberger-de Groot AC 1985 The role of the atrioventricular endocardial cushions in the septation of the heart. Int $\mathrm{J}$ Cardiol 8:25-44

15. Wenink ACG, Zevallos JC 1988 Developmental aspects of atrioventricular septal defects. Int J Cardiol 18:65-78

16. Van Gils FAW 1978 The formation of the atrioventricular heart valves. Acta Morphol Neerl-Scand 16:151(abstr)

17. Wenink ACG, Gittenberger-de Groot AC, Brom AG 1986 Developmental considerations of mitral valve anomalies. Int J Cardiol 11:85-98

18. De la Cruz MW, Gimenez-Ribotta M, Saravalli O, Cayre R 1983 The contribution of the inferior endocardial cushion on the atrioventricular canal to cardiac septation and to the development of the atrioventricular valves: study in the chick embryo. Am J Anat 166:63-72

19. Garcia-Pelaez I, Diaz-Gongora G, Martinez MA 1984 Contribution of the superior atrioventricular cushion to the left ventricular infundibulum. Acta Anat (Basel) 118:224-230

20. Kitten GT, Markwald RR, Bolender DL 1987 Distribution of basement membrane antigens in cryopreserved early embryonic hearts. Anat Rec 217:379-390
21. Mjaatvedt CH, Lepera RC, Markwald RR 1987 Myocardial specificity for initiating endothelial-mesenchymal cell transition in embryonic chick heart correlates with a particulate distribution of fibronectin. Dev Biol 119:59-67

22. Ffrench-Constant C, Hynes RO 1988 Patterns of fibronectin gene expression and splicing during cell migration in chicken embryos. Development 104:369-382

23. Ffrench-Constant C, Hynes RO 1989 Alternative splicing of fibronectin is temporally and spatially regulated in the chicken embryo. Development 106:375-388

24. Armstrong PB, Armstrong MT 1990 An instructive role for the interstitial matrix in tissue patterning: tissue segregation and intercellular invasion. $J$ Cell Biol 110:1439-1455

25. Ruzicka DL, Schwartz RJ 1988 Sequential activation of alpha-actin genes during avian cardiogenesis: vascular smooth muscle alpha-actin gene transcripts mark the onset of cardiomyocyte differentiation. J Cell Biol 107:25752586

26. Pexieder $T 1975$ Cell death in the morphogenesis and teratogenesis of the heart. Adv Anat Embryol Cell Biol 51:7-76

27. Hurle JM 1979 Scanning and light microscope studies of the development of the chick embryo semilunar heart valves. Anat Embryol (Berl) 157:69-80

28. Hurle JM, Ojeda JL 1979 Cell death during the development of the truncus and conus of the chick embryo heart. J Anat 129:427-439

29. Fananapazir K, Kaufman MH 1988 Observations on the development of the aortico-pulmonary spiral septum in the mouse. J Anat 158:157-172

30. Arguello C, De la Cruz MV, Sanchez C 1978 Ultrastructural and experimental evidence of myocardial cell differentiation into connective tissue cells in embryonic chick heart. J Mol Cell Cardiol 10:307-315 